\title{
Asociación entre el robo de néctar y las características florales en una comunidad montana de los Andes colombianos
}

\author{
S.V. Rojas-Nossa ${ }^{1, *}$ \\ (1) Departamento de Biología Vegetal y Ciencia del Suelo, Facultad de Ciencias, Universidad de Vigo, 36200 Vigo, España. \\ * Autor de correspondencia: S.V. Rojas-Nossa [srojas@uvigo.es]
}

> Recibido el 24 de abril de 2013, aceptado el 13 de julio de 2013.

Rojas-Nossa, S.V. (2013). Asociación entre el robo de néctar y las características florales en una comunidad montana de los Andes colombianos. Ecosistemas 22(2):107-112. Doi.: 10.7818/ECOS.2013.22-2.16

Los robadores de néctar son animales que usan una perforación en la corola para extraer el néctar de las flores. Este comportamiento puede generar cambios en diferentes direcciones y magnitudes sobre las diversas y complejas redes de polinización. No obstante, las consecuencias del robo de néctar sobre el funcionamiento de especies y comunidades neotropicales son prácticamente desconocidas. En este estudio se identificaron las aves paseriformes que se comportan como robadoras en ecosistemas del norte de los Andes en Colombia. Se cuantificó el robo de néctar en 38 especies de plantas y sus características florales asociadas a los visitantes florales. Seis especies de aves se comportaron como robadoras de néctar en el $60 \%$ de las plantas. Cinco especies de plantas con flores de corolas tubulares largas y abundante floración presentaron porcentajes de robo superiores al $20 \%$. Estos patrones de preferencia pueden asociarse con las altas recompensas energéticas producidas por flores de mayor tamaño y con una mayor eficiencia del forrajeo al visitar flores abundantes. Aunque preliminares, los resultados plantean un complejo escenario donde estas aves pueden ser potencialmente importantes para la evolución de los sistemas de polinización en el Neotrópico.

Palabras clave: colibrí, Conirostrum, Diglossa, interacción planta-animal, morfología floral, visitantes florales.

Rojas-Nossa, S.V. (2013). Association between nectar robbing and floral characteristics in a montane community of the Colombian Andes. Ecosistemas 22(2):107-112. Doi.: 10.7818/ECOS.2013.22-2.16

Nectar robbers are animals which use a perforation on the corolla in order to extract nectar from flowers. This behavior may generate changes of different directions and magnitudes on the diverse and complex pollination networks. However, the consequences of nectar robbing on the functioning of neotropical species and communities are practically unknown. Passeriform birds that behave as nectar robbers in ecosystems of northern Andes in Colombia were identified. Levels of nectar robbing in 38 plant species and their floral characteristics associated to floral visitors were characterized. Six bird species behave as nectar robbers in $60 \%$ of the plants. Five plant species with flowers of long tubular corollas and abundant flowers presented robbing percentages higher than $20 \%$. These preferences patterns can be associated with higher energy rewards produced by long flowers and a higher foraging efficiency when visiting abundant flowers. Besides preliminary, the results suggest a complex scenario where these birds could be potentially important for the evolution of pollination systems in the Neotropics.

Key words: hummingbird, Conirostrum, Diglossa, floral morphology, floral visitors, plant-animal interaction.

\section{Introducción}

La polinización de muchas plantas en el Neotrópico depende de las aves (Stiles 1981). Esta interacción cobra una mayor relevancia en las zonas altas de los Andes, en donde existen comunidades altamente diversas de aves nectarívoras, como los colibríes (Stiles 1985). Sin embargo, las ricas recompensas energéticas que producen las plantas son aprovechadas por animales que por su morfología no pueden acceder de manera legítima al néctar (es decir, por la abertura distal de la flor). En algunos casos, estos animales usan una perforación cerca de la base de la flor para extraer el néctar almacenado en el fondo de corolas tubulares o espolones. Este comportamiento se llama robo de néctar (Inouye 1980) y existen evidencias de sus efectos sobre la reproducción y la evolución de las plantas en regiones donde los robadores son principalmente insectos (Irwin et al. 2010).

Estudios en diferentes especies han demostrado que existe una relación entre la frecuencia de robo que experimentan las especies de plantas y determinadas variables asociadas con los visitantes florales. De manera que flores de corolas largas y mayor diámetro presentan una mayor frecuencia de robo (Castro et al. 2009, Navarro y Medel 2009). Además, en la mayoría de los casos, el robo de néctar causa una disminución en la producción de frutos y semillas, bien sea de manera directa (por territorialidad o daño a las estructuras reproductivas), o indirecta (generando cambios en el comportamiento de los visitantes legítimos) (Irwin et al. 2010). Considerando estas evidencias, se ha sugerido que los robadores de néctar pueden influir en la evolución de las plantas en sentidos diferentes a sus polinizadores (Navarro y Medel 2009). No obstante, desconocemos en gran medida los procesos ecológicos y evolutivos en los que potencialmente participan los robadores dentro una red compleja de polinización (Irwin et al. 2001).

Las aves del género Diglossa, constituyen algunos de los miembros más comunes de la avifauna de los Andes (Moynihan 1963). Estas aves paseriformes poseen características morfológicas, comportamentales y fisiológicas que les permiten ser roba- 
doras de néctar altamente especializadas (Villeumier 1969, Schondube y Martínez Del Río 2003, 2004). Se ha demostrado que su comportamiento de forrajeo tiene efectos sobre la reproducción de las plantas que usan como fuente de néctar (Arizmendi et al. 1996, Navarro 2001, Rojas-Nossa 2007, Navarro et al. 2008, Pelayo et. al 2011). Sin embargo, no existen estudios que estimen el porcentaje de robo de néctar a nivel de toda una comunidad de plantas. Los objetivos de esta investigación son identificar las especies de paseriformes que se comportan como robadoras de néctar en dos ecosistemas de alta montaña al norte de los Andes, estimar el porcentaje de robo de néctar que sufren las plantas nectaríferas de la comunidad y establecer la posible relación entre estos porcentajes con algunas características ecomorfológicas de las plantas.

\section{Material y métodos}

El estudio se llevó a cabo en dos fases: la primera entre marzo de 2003 y abril de 2004 y la segunda entre diciembre de 2011 y junio de 2012. Se realizó en los Cerros Orientales de Bogotá (4 $49^{\prime} 22^{\prime \prime} \mathrm{N}, 74^{\circ} 01^{\prime} 11,2$ "O), entre 2700 y 3150 msnm. El área presenta dos ecosistemas propios de regiones de alta montaña del norte de los Andes. El bosque altoandino se encuentra en las partes pendientes y húmedas de las montañas (Figs. 1a, b y c). El subpáramo consta de una densa vegetación de menor altura presente en pequeñas áreas de los filos de la montaña (Figs. 1d y e).

Para identificar las especies que actúan como robadoras de néctar se realizaron observaciones del comportamiento de las aves nectarívoras y conteos del número de individuos de cada especie en una banda de ancho infinita a lo largo de dos transectos en el bosque altoandino (de 1237 y $600 \mathrm{~m}$ de longitud) y dos en el subpáramo (de 1744 y 1200 m de longitud). Los horarios de observación de aves fueron de 06:00 - 12:00 durante 4 días cada 2 meses en la primera fase y 4 días en diciembre de 2011. Estos censos fueron realizados por dos observadores con la ayuda de binoculares $(8 \times 25)$. Se acumularon un total de 148 horas y 28 minutos de observación ( 88 horas y 43 minutos en el bosque altoandino y 59 horas y 45 minutos en el subpáramo). Del total acumulado de horas de observación 131 horas 5 minutos se realizaron en la primera campaña (2003-2004) y 17 horas y 23 minutos en la segunda (2011-2012). Con el fin de estimar el porcentaje de robo de néctar en las plantas nectaríferas de la comunidad, en estos mismos transectos se colectaron cada dos meses entre 10 y 50 flores de cada especie de planta nectarífera presente (4469 flores de 38 especies vegetales), y posteriormente se calculó el porcentaje de flores de cada especie que presentó una o más perforaciones hechas por robadores de néctar. Las diferencias en el número de flores colectadas para este análisis se debieron a la abundancia y accesibilidad de las flores en el área.

Entre marzo de 2003 a febrero de 2004 y abril a junio de 2012 se caracterizó una serie de atributos de las plantas relacionados con la interacción con los visitantes florales para un total de 38 especies pertenecientes a 16 familias. Tales atributos fueron: la presencia de estructuras de protección (como brácteas o sépalos alargados y/o gruesos), el tipo de corola (gamopétala o dialipétala), y el número de flores por hectárea. También se midió la producción de néctar en 24 horas (volumen y concentración de azúcares) y la morfología floral (longitud total de la corola, longitud del tubo o corola efectiva, diámetro de la corola en la entrada del tubo), en 30 flores por especie. Estas mediciones se realizaron en 2 a 10 flores por individuo dependiendo de la floración individual y poblacional en individuos escogidos aleatoriamente en el área de estudio.

Se realizó un ANOVA bifactorial con el fin de analizar las diferencias en la abundancia de las especies en el área y entre los dos hábitats estudiados, usando la medida de abundancia corregida por el tiempo de observación de cada especie en cada hábitat durante siete muestreos. Calculamos la correlación ordenada de Spearman con el fin de evaluar posibles asociaciones entre el porcentaje de
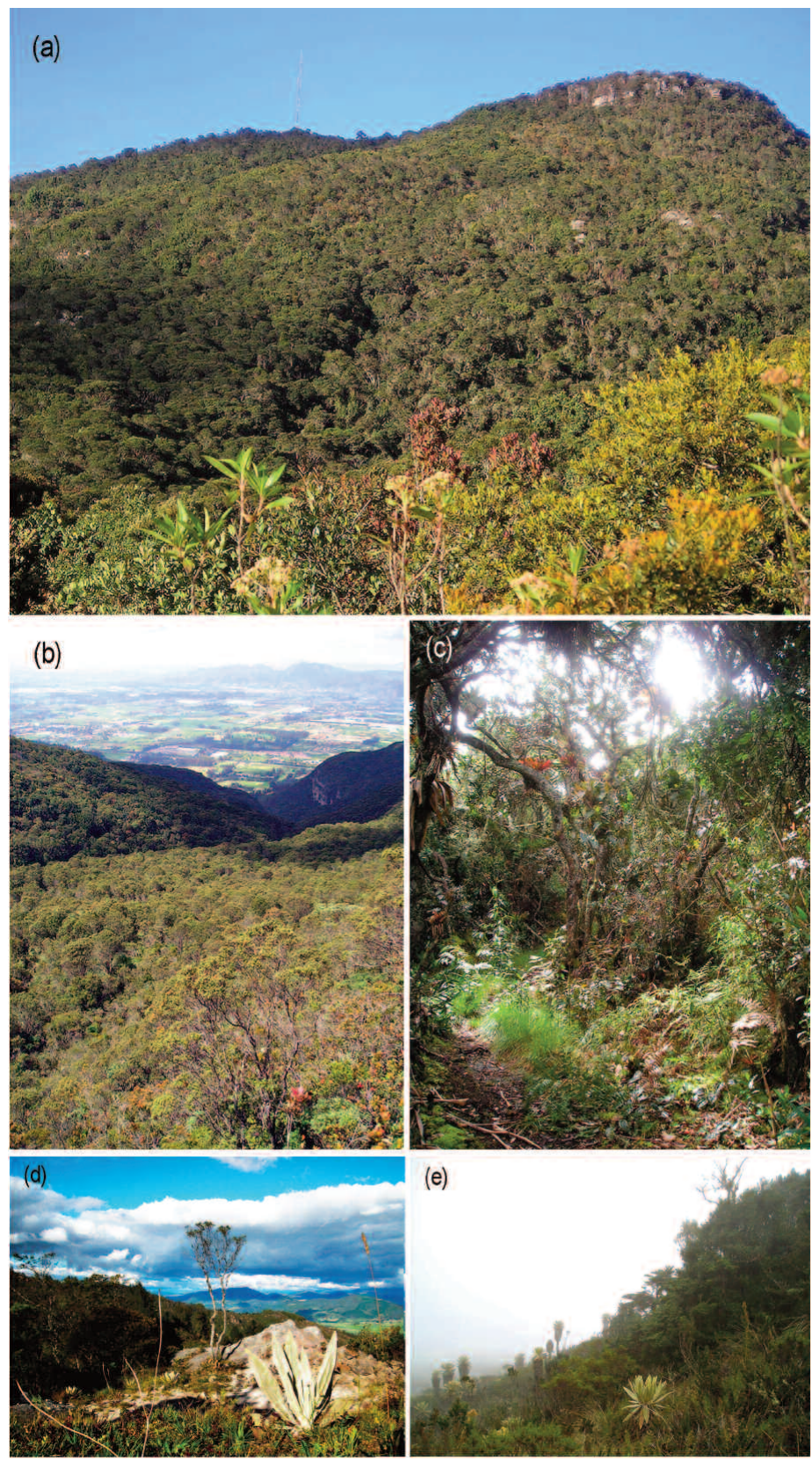

Figura 1. El sitio de estudio presenta dos de los ecosistemas típicos de alta montaña al norte de los Andes: El bosque altoandino (a) y (b) se presenta en las partes más húmedas y protegidas de la montaña. Allí se presenta una alta diversidad de plantas epífitas, como bromelias y orquídeas (c). El subpáramo (d) y (e) se encuentra en las partes secas, pendientes y rocosas de las montañas. Constituye una franja de vegetación de menor altura, con una mezcla de elementos del bosque y del páramo.

robo de las especies y las características de las plantas (solamente aquellas variables numéricas, tales como longitud total de la corola, longitud del tubo, diámetro, cantidad de flores por hectárea, volumen y concentración del néctar). Con el fin de modelar el efecto de la longitud de la corola efectiva y el número de flores por hectárea sobre el porcentaje de robo de néctar, se usó un modelo linear generalizado (MLG) con una estructura de error logística y la función log-link. La colinearidad entre estas variables no representó un problema en el modelo (máximo VIF=1.004). Los análisis fueron realizados en $\mathrm{R}$ versión 2.15.2.

\section{Resultados}

Seis especies de aves paseriformes se comportaron como robadoras de néctar: Conirostrum rufum, Diglossa caerulescens, $D$. cyanea, D. lafresnayii, D. humeralis, D. albilatera (Fig. 2). Todas ellas estuvieron presentes tanto en el bosque altoandino como en 


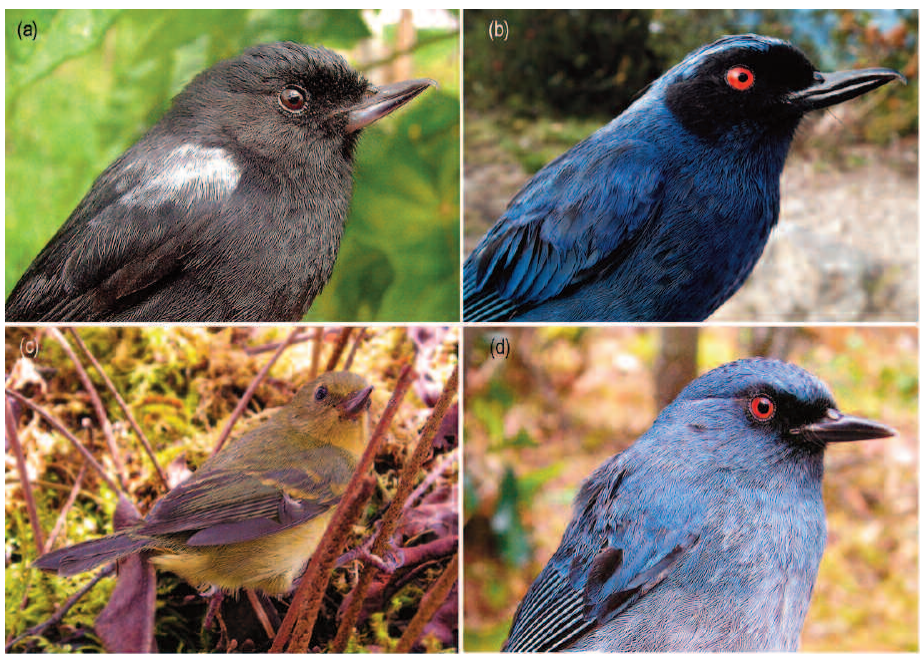

Figura 2. Especies comunes de Passeriformes robadoras de néctar de los ecosistemas altoandinos: (a) Diglossa humeralis, (b) D. cyanea, (c) D. albilatera hembra y $(d) \mathrm{D}$. caerulescens.

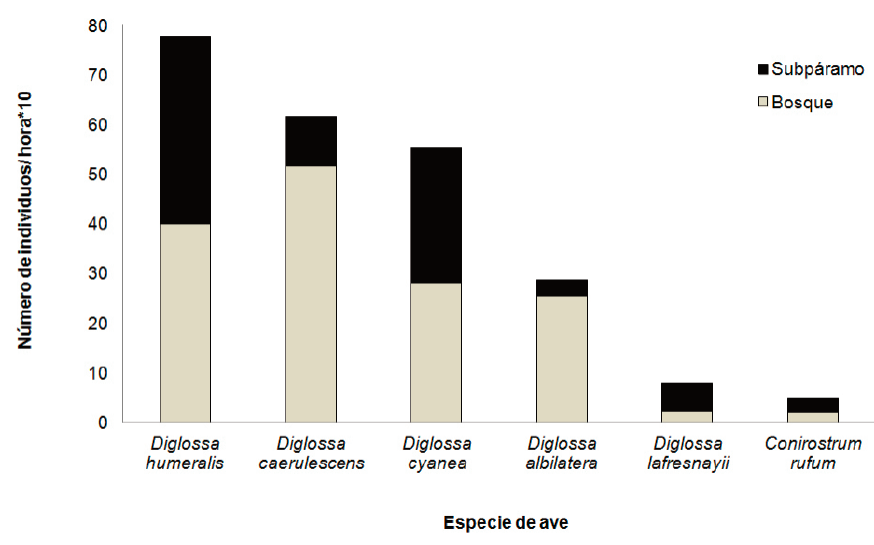

Figura 3. Abundancia de las especies de aves paseriformes que se comportan como robadoras de néctar en los ecosistemas de bosque altoandino y subpáramo del norte de los Andes.

el subpáramo (Fig. 3), aunque se encontraron diferencias significativas en su abundancia, tanto a nivel general en el área $\left(F_{5}, 83\right.$ $=3.69, \mathrm{P}=0.005$ ), como también en la abundancia dentro de los hábitats estudiados $\left(F_{5}, 83=2.55, P=0.035\right)$. Se registró un total de 315 individuos de estas especies. La especie más común en el área fue $D$. humeralis, ocurriendo con similares abundancias en los dos hábitats. $D$. caerulescens fue la especie más abundante en el bosque altoandino $y$, al igual que $D$. albilatera, forrajeó principalmente en los estratos altos de la vegetación. Estas aves no defendieron territorios de alimentación de visitantes florales legítimos, como los colibríes, quienes tuvieron aproximadamente el doble de abundancia (653 individuos) que los robadores en el mismo periodo y sitio de estudio. Por el contrario, algunas especies (como C. rufum, D. cyanea y D. caerulescens) presentaron con frecuencia complejos comportamientos sociales, como la participación en bandadas multiespecíficas mixtas para forrajear y defenderse de predadores.

A nivel de la comunidad de plantas, el porcentaje de robo de néctar presentó fluctuaciones a lo largo del año con un nivel mínimo (13.3\%) en mayo de 2003 y un máximo (47.1\%) en diciembre de 2003. No obstante, el porcentaje de robo estimado para el mismo mes en años diferentes fue muy similar (47.1 y 46.5 en diciembre de 2003 y 2011 respectivamente), reflejando poca variación de los niveles de robo de néctar entre años. Adicionalmente existió una variación relacionada con el hábitat, de manera que las flores presentes en el subpáramo presentaron más robo de néctar $(32.7 \%)$ que las flores del bosque altoandino (17.7 \%). Esta tendencia se mantuvo a lo largo del año, excepto en octubre de 2003, mes en el que se presentaron similares niveles de robo de néctar en los dos hábitats ( $35.8 \%$ en el bosque y $36.4 \%$ en el subpáramo).

El $60 \%$ de las especies de plantas estudiadas presentaron evidencias de robo de néctar (Fig. 4). Las plantas que experimentaron porcentajes de robo superiores al $20 \%$, presentaron síndrome de polinización por aves (sensu Faegri y van der Pijl 1979), como flores de color rojo y corolas tubulares largas (Fig. 5). Más de la mitad de las especies nectaríferas muestreadas de la comunidad presentaron un porcentaje de robo de néctar inferior al $5 \%$. Estas especies presentaron bajos números de flores por hectárea, flores de corola corta o dialipétala, y algunas especies tuvieron brácteas protegiendo la base o la totalidad de la corola.

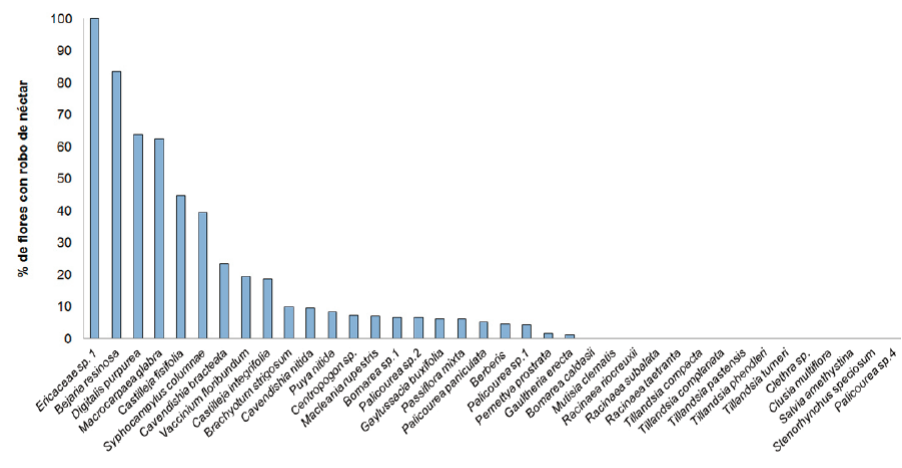

Figura 4. Robo de néctar en 38 especies presentes en los ecosistemas altoandinos. Las barras indican el porcentaje de flores con presencia de una o más perforaciones hechas por aves robadoras de néctar de una muestra de flores colectada aleatoriamente a lo largo de transectos.

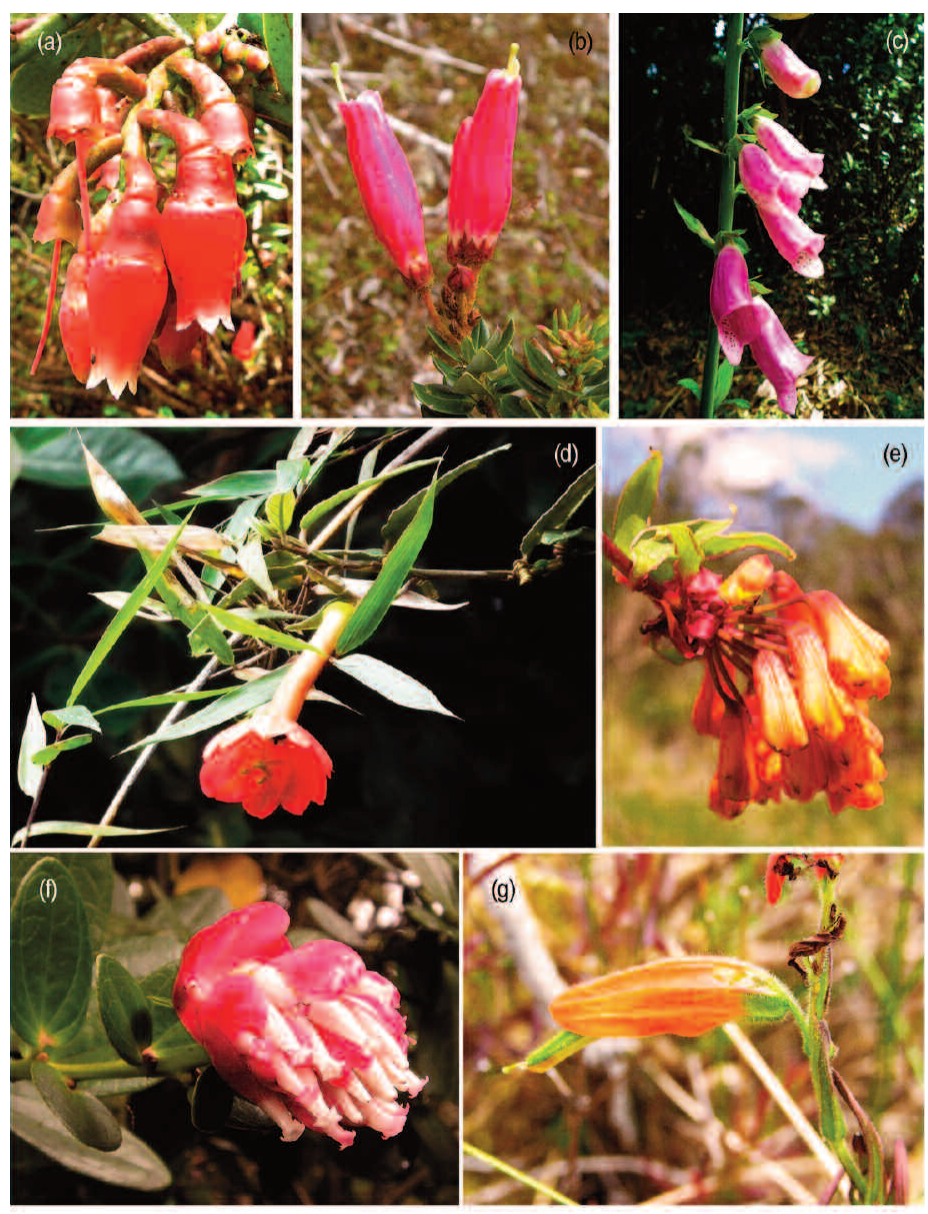

Figura 5. Plantas con corolas largas y abundante floración presentaron los mayores porcentajes de robo. Por ejemplo: (a) Macleania rupestris, (b) Bejaria resinosa, (c) Digitalis purpurea, (d) Passiflora sp., (e) Bomarea sp. 1, (f) Cavendishia nitida y (g) Castilleja fisifolia. 
Se encontraron correlaciones significativas entre el porcentaje de robo de néctar y la longitud de la corola efectiva $(\rho=0.38, P=$ $0.02, N=38$ ), y entre el robo de néctar y la cantidad de flores que produce la especie en el área $(\rho=0.57, \mathrm{P}<0.01, \mathrm{~N}=38)$. No se presentó una correlación significativa entre porcentaje de robo de néctar y la cantidad de néctar producida en 24 horas $(\rho=0.12, P=0.47$, $\mathrm{N}=38$ ) o entre éste y la concentración de azúcares en el néctar ( $\rho=$ - 0.24, $\mathrm{P}=0.15, \mathrm{~N}=36$ ). No obstante, la cantidad de néctar se correlacionó significativamente con la longitud total de la corola $(\rho=$ $0.64, \mathrm{P}<0.01, \mathrm{~N}=38$ ). Es decir que aquellas especies con flores de mayor longitud producen mayores cantidades de néctar.

Los análisis del MLG demostraron que las especies con flores de corolas tubulares más largas y con abundante floración presentaron mayor incidencia de robo de néctar (Fig. 6). Estas variables influyeron significativamente en la probabilidad de que las especies sufran robo de néctar (coeficiente $\pm \mathrm{DE}=1.63 \pm 0.07, \mathrm{p}<0.001$ para la longitud efectiva de la corola y coeficiente $\pm \mathrm{DE}=1.04 \pm 0.07$, $\mathrm{p}<0.001$ para la cantidad de flores por hectárea).

\section{Discusión}

La diversidad de aves paseriformes que se comportan como robadoras de néctar es mayor de la que había sido reportada en otras localidades (Arizmendi et al. 1996, Navarro 1999, Navarro et al. 2008). Considerando que en ambientes similares se ha registrado una mayor importancia relativa en la tasa de visitas por aves robadoras de néctar en comparación con la tasa de robo de néctar por insectos (Navarro 1999, Navarro et al. 2008), probablemente las aves constituyen los robadores de néctar más importantes en ambientes montanos de los Andes. De hecho, todas las especies del género Diglossa son conocidas por comportarse como robadoras de néctar en flores polinizadas por colibríes (Villeumier 1969, Navarro 2001), aunque este estudio constituye el primer registro de Conirostrum rufum (especie casi endémica de Colombia, según BirdLife International 2013), como robador de néctar.

Se presentaron diferencias temporales (a lo largo de un año) y espaciales (entre los dos hábitats estudiados) en las abundancias poblacionales de estas aves y en la incidencia de robo de néctar en la comunidad de plantas. Este tipo de dinámica a nivel comunitario es frecuente en las interacciones de polinización en ecosistemas Neotropicales (Stiles 1985, Arizmendi 2001, Gutiérrez et al. 2004). Además de los factores biológicos propios de las poblaciones y las comunidades, estos cambios en las abundancias podrían deberse a movimientos poblacionales generados por diferencias en los requerimientos energéticos de las aves combinados con va- riaciones en la disponibilidad de recursos en el ambiente (Stiles 1975, 1985, Gutiérrez et al. 2004). El hecho de que tanto las abundancias poblacionales de las aves como los porcentajes de robo de néctar detectados en este estudio presentaron fluctuaciones temporales a lo largo de un año (pero no entre años), puede constituir una evidencia de que el robo de néctar por aves paseriformes es un fenómeno permanente en estos ecosistemas, aunque presenta una dinámica temporal y espacial que debe ser tenida en cuenta en futuras investigaciones.

Además de las especies de aves nectarívoras paseriformes registradas, en los ecosistemas andinos habita una gran diversidad de colibríes que presentan interacciones con comunidades de plantas igualmente diversas (Wolf et al. 1976, Snow y Snow 1980, Gutiérrez et al. 2004). Los dos grupos de aves (tanto los colibríes que realizan visitas legítimas, como los robadores de néctar de los géneros Diglossa y Conirostrum) consumen néctar de las mismas especies de plantas (Gutiérrez-Zamora 2008, Rojas-Nossa 2007), por lo que podría esperarse que se presentara una fuerte competencia por este recurso. Sin embargo no se observaron comportamientos agonísticos, como defensa de territorios de alimentación, por parte de las especies robadoras hacia otras aves. De manera que probablemente estos robadores de néctar no ejercen un efecto directo sobre la accesibilidad de los recursos de néctar para otras aves nectarívoras en esta localidad. Sin embargo, en este caso podría estar ocurriendo competencia por explotación del néctar como recurso, de manera que el forrajeo de las aves robadoras podría tener un efecto indirecto sobre los sistemas de polinización al disminuir la cantidad de néctar disponible en el ambiente (ver Maloof e Inouye 2000). Adicionalmente, dada la alta abundancia de robadores de néctar en relación a los colibríes en la comunidad de aves estudiada y su presencia en diferentes hábitats a lo largo de la cordillera de los Andes (Moynihan 1963, Villeumier 1969), es necesario considerar a estos organismos como actores importantes en las interacciones de polinización y evolución los sistemas ave-planta de los ecosistemas montanos Neotropicales. Sin embargo, aún requieren ser exploradas tanto la magnitud como la dirección de los efectos del robo de néctar para los polinizadores y la reproducción de las plantas.

En la comunidad de plantas estudiada, la longitud de la corola se correlacionó positivamente con el porcentaje de robo de néctar. Este mismo patrón fue observado en otras plantas polinizadas por aves en las cuales los robadores de néctar fueron colibríes (Lara y Ornelas 2001) o himenópteros (Urcelay et al. 2006, Castro et al. 2009, Navarro y Medel 2009). Este resultado implica procesos relacionados con las características morfológicas y conductuales de
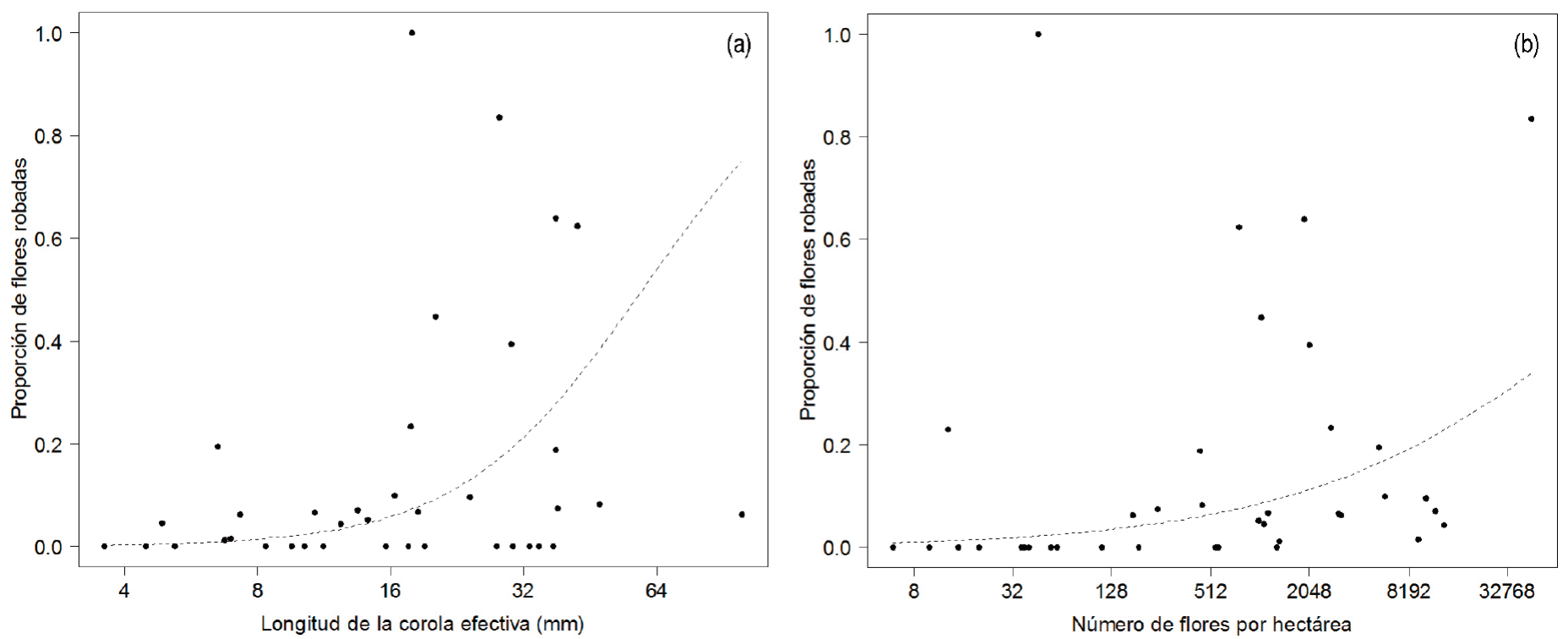

Figura 6. Asociación entre la longitud de la corola efectiva (a) y el número de flores por hectárea (b) con la frecuencia de robo en 38 especies de plantas del norte de los Andes. La línea representa el modelo ajustado. 
las aves que actúan como robadoras de néctar. Por una parte, algunas especies del género Diglossa realizan con frecuencia visitas legítimas en flores péndulas de corolas medianas y cortas (Rojas-Nossa 2007). No obstante, estas aves realizan robo de néctar en flores con corolas tubulares con una longitud mayor a su pico. Por lo tanto, otro factor adicional debería ser responsable del patrón de preferencias de estas aves hacia plantas con flores largas observado en la presente investigación. Al igual que en otros estudios, se registró una relación positiva entre la longitud total de la corola y la cantidad de néctar producido por las flores (Castro et al. 2009, Navarro y Medel 2009). Se ha demostrado que las flores más largas tienen nectarios más grandes y por lo tanto contienen mayores cantidades de néctar (Ornelas et al. 2007, Gómez et al. 2008). A su vez, los robadores de néctar tienen la habilidad de asociar la longitud de las flores con las recompensas energéticas que contienen y prefieren visitar flores con mayores recompensas. Como resultado, los robadores de néctar visitan con más frecuencia flores de corolas largas. Adicionalmente, la cantidad de flores disponible en un área puede actuar como un atrayente para los visitantes florales, ya que probablemente el hecho de que un recurso sea más abundante implica que es más fácilmente detectable (Eckhart 1991, Mitchell et al. 2004). Por lo tanto el esfuerzo de búsqueda durante el forrajeo se minimiza, aumentando así la eficiencia para la obtención de los recursos alimenticios. Estos dos factores combinados, una mayor recompensa energética en flores de corolas largas y una mayor eficiencia para detectar las flores más abundantes, favorece una mayor preferencia de estas aves para alimentarse en plantas con corolas largas y abundante floración.

Se ha sugerido que tales patrones de preferencias por parte de los robadores, sumado al hecho de que estos pueden modificar el éxito reproductivo de las plantas robadas, pueden ejercer presiones selectivas sobre determinadas características de las plantas (Irwin 2006). Sin embargo, las evidencias sobre las consecuencias del robo de néctar por paseriformes son contrastantes. En determinados casos el forrajeo de las especies de Diglossa puede ser positivo para las plantas, ya que pueden actuar como polinizadoras (Graves 1982, Arizmendi et al. 1996, Rojas-Nossa 2007), o contribuyen indirectamente al flujo de polen (Pelayo et al. 2011). Por el contrario, es negativo cuando las flores robadas reciben menores cantidades de polen en el estigma ( $\mathrm{Na}-$ varro et al. 2008), o cuando el excesivo gasto energético para reemplazar el néctar consumido por los robadores causa una disminución en la fructificación de las plantas (Navarro 2001). Dados los altos porcentajes de robo de néctar que experimentan algunas especies de plantas de la comunidad estudiada y la gran diversidad y especialización de los robadores de néctar en los ecosistemas de alta montaña Neotropicales, estos resultados plantean un complejo escenario donde estas aves son potencialmente importantes para la evolución de algunas las características de las plantas y las especies que interactúan con ellas. En futuras investigaciones es necesario continuar el estudio de este fenómeno a diferentes escalas ecológicas, temporales y espaciales que permitan explorar las consecuencias del robo de néctar para la evolución de los sistemas de polinización en regiones megadiversas, como los Andes tropicales.

\section{Conclusiones}

1 En los ecosistemas de alta montaña del norte de los Andes las especies de aves paseriformes de los géneros Diglossa y Conirostrum son los principales robadores de néctar.

2 En estas comunidades vegetales las especies de corolas tubulares largas y abundante producción de flores presentan una mayor incidencia de robo de néctar. Estos resultados pueden asociarse con las preferencias de las aves hacia las altas recompensas energéticas producidas por flores de mayor tamaño y con una mayor eficiencia de forrajeo en flores abundantes.

\section{Agradecimientos}

G. Stiles, L. Navarro y A. Gutiérrez contribuyeron significativamente en el planteamiento y desarrollo de esta investigación. S. Nossa, B. Rojas, F. Nossa, A. Rico, C. Sandoval, E. Carrillo, P. Velasco, J. Posada, A. Pinto, O. Acevedo y otros miembros del GOUN prestaron una ayuda invaluable durante el trabajo de campo. A D. Rojas por la asesoría estadística. A los habitantes y trabajadores del Conjunto Residencial Bosques de Torca y Floresta de la Sabana por su colaboración para acceder a las zonas de estudio. La primera fase de esta investigación fue realizada gracias a la Beca Alexander y Pamela Skutch (AFO), Ecotono y la Universidad Nacional de Colombia. La segunda fase fue realizada con el apoyo de la Asociación Española de Ecología Terrestre, la Universidad de Vigo y la Universidad Nacional de Colombia. A la editora asociada responsable y los dos revisores anónimos por las valiosas sugerencias que contribuyeron a mejorar la calidad del manuscrito.

\section{Referencias}

Arizmendi, M.C., Domínguez, C.A., Dirzo, R. 1996. The role of an avian nectar robber and of hummingbird pollinators in the reproduction of two plant species. Functional Ecology 10:119-127.

Arizmendi, M.C. 2001. Multiple ecological interactions: Nectar robbers and hummingbirds in a highland forest in Mexico. Canadian Journal of Zoology 79:997-1006.

BirdLife International 2013. Informativa de especie: Conirostrum rufum. Obtenido de: http://www.birdlife.org en 10/07/2013.

Castro, S., Silveira, P., Navarro, L. 2009. Floral traits variation, pollinator attraction and nectar robbers in Polygala vayredae (Polygalaceae). Ecological Research 24:47-55.

Eckhart, V.M. 1991. The effects of floral display on pollinator visitation vary among populations of Phacelia linearis (Hydrophyllaceae). Ecology and Evolution 5:370-384

Faegri K., van der Pijl L. 1979. The Principles of Pollination Ecology. Pergamon, Oxford, Reino Unido.

Gómez, J.M., Bosch, J., Perfectti, F., Fernández, J.D., Abdelaziz, M., Camacho, J.P.M. 2008. Association between floral traits and rewards in Erysimum mediohispanicum (Brassicaceae). Annals of Botany 101:1413-1420.

Graves, G. 1982. Pollinaton of a Tristerix mistletoe (Loranthaceae) by Diglossa (Aves, Thraupidae). Biotropica 14:316-317

Gutiérrez, A., Rojas-Nossa, S.V., Stiles, F.G. 2004. Dinámica anual de la interacción colibrí-flor en ecosistemas Altoandinos. Ornitología neotropical 15:205-213.

Gutiérrez-Zamora, A. 2008. Las interacciones ecológicas y estructura de una comunidad altoandina de colibríes y flores en la Cordillera Oriental de Colombia. Ornitología Colombiana 7:17-42.

Inouye, D.W. 1980. The terminology of floral larceny. Ecology 61:1251-1253.

Irwin, R.E., Bronstein, J.L., Manson, J.S., Richardson, L. 2010. Nectar Robbing: Ecological and Evolutionary Perspectives. Annual Review of Ecology, Evolution, and Systematics 41:271-92.

Irwin, R.E. 2006. The consequences of direct versus indirect species interactions to selection on traits: pollination and nectar robbing in Ipomopsis aggregata. American Naturalist 167:315-328.

Irwin, R.E., Brody, A.K., Waser, N.M. 2001. The impact of floral larceny on individuals, populations, and communities. Oecologia 129:161-168.

Lara, C., Ornelas, J.F. 2001. Preferential nectar robbing of flowers with long corollas: experimental studies of two hummingbird species visiting three plant species. Oecologia 128:263-273.

Mitchell, R.J., Karron, J.D., Holmquist, K.G., Bell, J.M. 2004. The influence of Mimulus ringens floral display size on pollinator visitation patterns. Functional Ecology 18:116-124.

Maloof, J.E., Inouye D.W. 2000. Are nectar robbers cheaters or mutualists? Ecology 81: 2651-61.

Moynihan, M. 1963. Inter-specific relations between some Andean birds. Ibis. 105:327-339.

Navarro, L. 1999. Pollination ecology and effect of nectar removal in Macleania bullata (Ericaceae). Biotropica 31:618-625.

Navarro, L. 2001. Reproductive biology and effect of nectar robbing on fruit production in Macleania bullata (Ericaceae). Plant Ecolology 152:59-65. 
Navarro, L., Guitián, P., Ayensa, G. 2008. Pollination ecology of Disterigma stereophyllum (Ericaceae) in Southwestern Colombia. Plant Biology 10:512-518.

Navarro L, Medel R. 2009. Relationship between floral tube length and nectar robbing in Duranta erecta L. (Verbenaceae). Biological Journal of the Linnean Society 96:392-398.

Ornelas, J.F., Ordano, M., de Nova, A.J., Quintero, M.E., Garland, T. Jr 2007. Phylogenetic analysis of interspecific variation in nectar of hummingbird-visited plants. Journal of Evolutionary Biology 20:1904-1917.

Pelayo, R.C., Rengifo, C., Soriano, P.J. 2011. Avian nectar robbers of Passiflora mixta (Passifloraceae): Do they have a positive effect on the plant? Interciencia 36:587-592.

Rojas-Nossa, S.V. 2007. Estrategias de extracción de néctar por pinchaflores (Aves: Diglossa y Diglossopis) y sus efectos sobre la polinización de plantas de los altos Andes. Ornitología Colombiana 5:21-39.

Schondube, J.E., Martínez Del Río, C. 2003. The flowerpiercers' hook: an experimental test of an evolutionary trade-off. Proceedings of the Royal Society B: Biological Sciences 270:195-198.

Schondube, J.E., Martínez Del Río, C. 2004. Sugar and protein digestion in Flowerpiercers and Hummingbirds: A comparative test of adaptive convergence. Journal of Comparative Physiology B 174:263-273.
Snow, D.W., Snow, B.K. 1980. Relationships between hummingbirds and flowers in the Andes of Colombia. Bulletin of the British Museum (Natural History), Zoology series 38:105-139.

Stiles, F.G. 1975. Ecology, flowering phenology, and hummingbird pollination of some Costa Rican Heliconia species. Ecology 56:285-301.

Stiles, F.G. 1981. Geographical aspects of birdflower coevolution, with particular reference to Central America. Annals of the Missouri Botanical Garden 68:323-351.

Stiles, F.G. 1985. Seasonal patterns and coevolution in the hummingbirdflower community of a Costa Rican Subtropical forest. Ornithological Monographs 36:757-787.

Urcelay, C., Morales, C.L., Chalcoff, V.R. 2006. Relationship between corolla length and floral larceny in the South American hummingbird-pollinated Campsidium valdivianum (Bignoniaceae). Annales Botanici Fennici. 43:205-211.

Villeumier, F. 1969. Systematics and evolution in Diglossa (Aves: Coerebidae). A.M.N.H. Novitates 2831.

Wolf, L.L., Stiles, F.G., Hainsworth, F.R. 1976. Ecological organization of a tropical highland hummingbird community. Journal of Animal Ecology 32:349-379. 